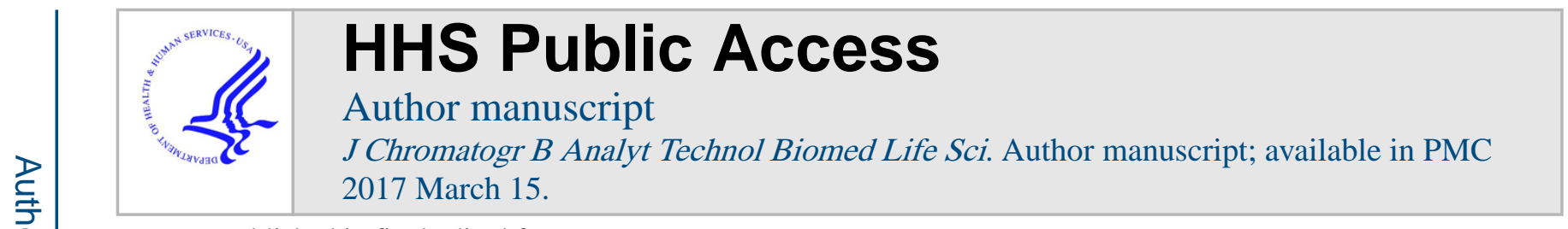

Published in final edited form as:

J Chromatogr B Analyt Technol Biomed Life Sci. 2016 March 15; 1015-1016: 34-41. doi:10.1016/

j.jchromb.2016.02.012.

\title{
Development and validation of an UPLC-MS/MS method for the quantification of irinotecan, $\mathrm{SN} 38$ and $\mathrm{SN}-38$ glucuronide in plasma, urine, feces, liver and kidney: Application to a pharmacokinetic study of irinotecan in rats
}

\author{
Sumit Basu ${ }^{1,2}$, Min Zeng ${ }^{1,3}$, Taijun Yin ${ }^{1}$, Song Gao ${ }^{1}$, and Ming $\mathrm{Hu}^{1, *}$ \\ ${ }^{1}$ Department of Pharmacological and Pharmaceutical Sciences, College of Pharmacy, University \\ of Houston, 1441 Moursund Street, Houston, TX, 77030, USA \\ ${ }^{2}$ Center for Pharmacometrics and Systems Pharmacology, College of Pharmacy, University of \\ Florida, 6550 Sanger Road, Orlando, FL, 32827, USA \\ ${ }^{3}$ Department of Thoracic and Cardiomacrovascular surgery, Shiyan Taihe Hospital Affiliated to \\ Hubei University of Medicine, Shiyan, Hubei 442000, China
}

\begin{abstract}
The objective of this research is to develop and validate a sensitive and reproducible UPLCMS/MS method to quantify irinotecan, its active metabolite SN-38 and SN-38 glucuronide (phase II metabolite of SN-38) simultaneously in different bio-matrices (plasma, urine, feces), tissues (liver and kidney) and to use the method to investigate its pharmacokinetic behavior in rats. Irinotecan, $\mathrm{SN}-38$ and $\mathrm{SN}-38$ glucuronide has been resolved and separated by $\mathrm{C} 18$ column using acetonitrile and $0.1 \%$ formic acid in water used as the mobile phases. Triple quadruple mass spectrometer using multiple reaction monitoring (MRM) with positive scan mode were employed to perform mass analysis. The results showed that the linear response range of irinotecan and $\mathrm{SN}-38$ in plasma, feces, liver and kidney is $4.88-10000 \mathrm{nM}, 39-5000 \mathrm{nM}, 48.8-6250 \mathrm{nM}$ and $48.8-6250 \mathrm{nM}$, respectively $\left(\mathrm{R}^{2}>0.99\right)$. In case of SN-38 glucuronide, the standard curves were linear in the concentration range of $6.25-2000 \mathrm{nM}, 4.88-1250 \mathrm{nM}, 9.8-1250 \mathrm{nM}$ and $9.8-$ $1250 \mathrm{nM}$ in plasma, feces, liver and kidney homogenates, respectively. The lower limit of detection (LLOD) of irinotecan, SN-38 and SN-38 glucuronide was determined to be less than 25 $\mathrm{nM}$ in all bio-matrices as well as tissue homogenates. Recoveries of irinotecan, SN-38 and SN-38 glucuronide at three different concentrations (low, medium and high) were not less than $85 \%$ at three different concentrations in plasma and feces. The percentage matrix factors in different biomatrices and tissues were within $20 \%$. The UPLC-MS/MS method was validated with intra-day and inter-day precision of less than $15 \%$ in plasma, feces, liver and kidney. Owing to the high sensitivity of this method, only $20 \mu \mathrm{l}$ of plasma, urine and homogenates of liver, kidney and feces is needed. The validated method has been successfully employed for pharmacokinetic evaluation
\end{abstract}

\footnotetext{
*Author to whom correspondence should be addressed [Tel: (713) 795-8320; Fax: (713) 795-8305. mhu@uh.edu].

Publisher's Disclaimer: This is a PDF file of an unedited manuscript that has been accepted for publication. As a service to our customers we are providing this early version of the manuscript. The manuscript will undergo copyediting, typesetting, and review of the resulting proof before it is published in its final citable form. Please note that during the production process errors may be discovered which could affect the content, and all legal disclaimers that apply to the journal pertain.
} 
of irinotecan in male wistar rats to quantify irinotecan, $\mathrm{SN}-38$ and $\mathrm{SN}-38$ glucuronide in plasma, feces, and urine samples.

\section{Keywords}

Irinotecan; SN-38; Pharmacokinetics; UPLC-MS/MS; SN-38 glucuronide

\section{Introduction}

Irinotecan (7-ethyl-10-[4-(1-piperidino)-1-piperidino]-carbonyloxycamptothecine, CPT-11, Fig. $1 \mathrm{~B}$ ) is an important component of FOLFIRI and FOLFIRINOX chemotherapy regimen which are used as first line treatment against colorectal and advanced pancreatic cancer $[1$, 2]. It has also shown clinical activity against other types of cancers such as lung, ovarian, cervical, gastric, refractory lymphoma and leukemia etc. [3-5]. Irinotecan is a pentacyclic semisynthetic derivative of camptothecin which is isolated from the bark of a tree named Camptotheca acuminate in 1966 [6, 7]. Irinotecan, through the action of liver carboxylesterase gets converted into its active metabolite SN-38 (7-ethyl-10hydroxycamptothecin, Fig. $1 \mathrm{C}$ ) which is 100-1000 times as potent as irinotecan [8,9]. Anticancer activity of irinotecan is attributed to the inhibitory effect of SN-38 on DNA topoisomerase I which plays an important role in DNA replication and transcription [10, 11]. Formation of an irreversible complex of SN-38, DNA topoisomerase I enzyme and the ligated DNA strand leads to the breakage of double stranded DNA which ultimately causes cell death. Owing to the poor solubility of SN-38, irinotecan is currently used as a prodrug in clinical applications.

Irinotecan exhibits a complicated metabolic profile by interacting with various drug metabolizing enzymes as well as efflux transporters. Studies indicated that both biliary as well as intestinal excretion of irinotecan and its metabolites are regarded as significant contributing factors to elevate the colonic SN-38 content which causes late onset diarrhea [12-16]. In spite of knowing the fact that predominant efflux transporters (P-gp and MRP2) mediate the excretion of irinotecan and its metabolites, the role of efflux transporters on the disposition of irinotecan and its metabolites at different metabolic organs (liver, intestine and kidney) has not been clearly defined till date. Quantification of irinotecan and its metabolites in different bio-matrices (bile, plasma, intestinal perfusate and urine) as well as in different metabolic organs (liver and kidney) will allow us to elucidate the role of predominant efflux transporters on the disposition and the intestinal toxicity of irinotecan.

Generally, inactivation of SN-38 to its phase II metabolite SN-38 glucuronide (Fig. 1 D) occurs through an enzymatic reaction mediated by the UDP-glucuronosyltransferase 1A1 isoform (UGT1A1) [17, 18]. UGT1A1 plays an important role in the disposition as well as in the intestinal toxicity of irinotecan. In the colon, SN-38 glucuronide, after interacting with the bacterial $\beta$ glucuronidase enzyme secreted from commensal microbiota, can regenerate toxic SN-38 which has the potential to kill the normal cells in the intestine resulting in late onset diarrhea [19]. At present, several compounds are being synthesized which can predominantly inhibit the activity of the bacterial $\beta$-glucuronidase resulting in the reduction 
of the generation of SN-38 from inactive SN-38 glucuronide [20]. In addition, preclinical studies indicated that around 2 to $22 \%$ of SN-38 glucuronide was excreted into the bile over 24 hours which was comparatively higher than biliary excretion of SN-38 (7 - 9\%) [15, 21]. Similarly clinical study of irinotecan also indicated that around $3 \%$ of dose is excreted through urine [12]. So in this regard, direct quantification of SN-38 glucuronide in different biomatrices as well as in major metabolic organs will enable us to get the holistic idea of the disposition of irinotecan.

It has been shown that the intact lactone ring of camptothecin (CPT) derivative is an important factor to retain its anticancer activity $[10,11,22]$. However, the lactone ring of CPT derivatives undergoes a $\mathrm{pH}$ sensitive, reversible hydrolysis and converts into carboxylate form at physiologic $\mathrm{pH}$ [23]. Boyd et al. showed that $\mathrm{pH}$ played an important role in the inter conversion of lactone and carboxylate forms of CPT in the solution. Experiments showed that at $\mathrm{pH} 3$ to 5, lactone is the most stable form of CPT, whereas at $\mathrm{pH}$ 9, it rapidly converts into carboxylate form [24]. Till date, HPLC methods coupled with fluorescence detectors have been the most predominant method for detection of CPT derivatives as they can detect both lactone and carboxylate forms in different biological matrices.

Different bioanalytical techniques, mostly based on reverse phase liquid chromatography coupled with fluorescence detection, have been proposed to determine irinotecan and SN-38 in various biological matrices. However, these fluorescence methods suffered from several drawbacks such as lengthy preparation time, limited sensitivity and high sample volumes [25-27]. Recently, liquid chromatography coupled with mass spectrometry (LC-MS and LCMS/MS) has become one of the preferred analytical tools for the rapid and efficient quantification of small and large molecules in different biological matrices due to the unique combination of high specificity, sensitivity and high sample throughput possibilities.

Although several LC-MS/MS methods have been developed and implemented for the quantification of irinotecan and SN-38 in rabbit, mouse and human plasma, very few of them developed a UPLC-MS/MS method of SN-38 glucuronide [28-31]. Similarly, though quantification of irinotecan and SN-38 was done in mouse tissues (brain, liver, kidney etc.), there was no report of the quantification of SN-38 glucuronide in these tissues $[32,33]$. Also, to the best of our knowledge there is no LC-MS/MS method available to quantify irinotecan, SN-38 and SN-38 glucuronide simultaneously in urine and feces. In this study, we developed and validated a simple, rapid and sensitive LC-MS/MS method for simultaneous quantification of total concentration of irinotecan, SN-38 and SN-38 glucuronide in rat plasma, feces, urine, liver and kidney homogenate to support pharmacokinetic studies in rats.

\section{Experimental}

\subsection{Chemicals and reagents}

Irinotecan, SN-38, CPT, uridine-5' -diphosphate- $\beta$,D-glucuronic acid ester (UDPGA), Dsaccharic-1,4-lactone monohydrate, magnesium chloride, Hanks' balanced salt solution (powder form) and formic acid were purchased from Sigma-Aldrich (St. Louis, MO, USA). Expressed human UGT isoforms (UGT1A1) was purchased from BD Biosciences (Woburn, 
MA, USA). Solid phase extraction (C18) columns were purchased from J.T. Baker (Phillipsburg, NJ, USA). Acetonitrile, methanol and water (LC-MS grade) were purchased from EMD (Gibbstown, NJ, USA). Water was deionized by a Milli-Q water purification system of Millipore (Bedford, MA, USA). Intravenous irinotecan hydrochloride injection (20 mg/ml) was purchased from Teva Pharmaceuticals (Pearl River, NY, USA).

2.1.1 Bio Synthesis of SN-38 glucuronide- $\mathrm{SN}-38$ glucuronide was biosynthesized using human expressed UGTs (UGT1A1 isoform). In this study, SN-38 was incubated with UGT1A1 for 24 hours which resulted in the formation of $95.8 \% \mathrm{SN}-38$ glucuronide. Then, the mixture containing both $\mathrm{SN}-38$ and $\mathrm{SN}-38$ glucuronide was extracted with a liquid-liquid extraction method using dichloromethane (DCM) as the organic solvent to remove SN-38. The resulting aqueous layer of SN-38 glucuronide was collected, and each $5 \mathrm{ml}$ of the collected layer were applied to a $\mathrm{C} 18$ solid phase extraction column. After washing out the salt, $1 \mathrm{ml}$ of methanol was used to elute $\mathrm{SN}-38$ glucuronide. The eluted methanol fractions from multiple extractions were pooled and air dried, and the residue was reconstituted with $100 \mu \mathrm{l}$ of 50\% methanol-water to concentrate SN-38 glucuronide. In each step, the respective peak area of SN-38 and SN-38 glucuronide were measured in UPLC using PDA detector at wavelength of 375.2 and $380 \mathrm{~nm}$ respectively, to calculate the purity of SN-38 glucuronide in the mixture. Apart from UPLC quantification, MS/MS spectrum of SN-38 glucuronide was also analyzed to confirm its presence in the solution.

\subsection{Instruments and Conditions}

2.2.1. UPLC - The chromatographic separation of irinotecan, $\mathrm{SN}-38$ and SN-38 glucuronide was achieved by a Waters Acquity ${ }^{\mathrm{TM}}$ UPLC H-Class system with a diode array detector (DAD) and a flow-through-needle sample manager. Analysis was carried out using Acquity UPLC BEH C18 Column $\left(2.1 \mathrm{~mm} \times 50 \mathrm{~mm}, 300^{\circ} \mathrm{A}, 1.7 \mu \mathrm{m}\right.$, Waters, Milford, MA, USA). Mobile phase A ( $0.1 \%$ formic acid in water [v/v]) and mobile phase B ( $100 \%$ acetonitrile) were operated with a gradient elution at a flow rate of $0.4 \mathrm{ml} / \mathrm{min}$ as follows: $10 \% \mathrm{~B} \rightarrow 25 \% \mathrm{~B}(0-0.5 \mathrm{~min}), 25 \% \mathrm{~B} \rightarrow 40 \% \mathrm{~B}(0.5-1 \mathrm{~min}), 40 \% \mathrm{~B}(1-2.5 \mathrm{~min}), 40 \%$ $\mathrm{B} \rightarrow 10 \% \mathrm{~B}(2.5-4.5 \mathrm{~min})$. The column temperature and sample temperature was $60^{\circ} \mathrm{C}$ and $20^{\circ} \mathrm{C}$. The injection volume was $10 \mu \mathrm{l} .100 \mathrm{nM}$ camptothecin (CPT; Fig. $1 \mathrm{E}$ ) in $50 \%$ methanol-water was used as internal standard (IS).

2.2.2. Mass Spectrometry-LC-MS/MS analysis for irinotecan, $\mathrm{SN}-38$ and $\mathrm{SN}-38$ glucuronide was performed with an API 5500 Qtrap triple quadruple mass spectrometer coupled with a TurbolonSpray ${ }^{\mathrm{TM}}$ (Applied Biosystem- MDS SCIEX, Framingham, MA, USA). The system was operated in positive electrospray ionization (ESI) and multiple reactions monitoring (MRM) scan mode. All data were acquired and processed using Analyst ${ }^{\circledR} 1.5 .2$ software with hotfixes (AB SCIEX).

\subsection{Method validation}

2.3.1. Calibration curve and LLOD-Calibration standards were prepared in $50 \%$ aqueous methanolic solution by diluting a stock solution of irinotecan, $\mathrm{SN}-38$ and $\mathrm{SN}-38$ glucuronide to final concentrations of 50,50 and $10 \mu \mathrm{M}$ respectively and were kept at $-80^{\circ} \mathrm{C}$. Working solutions were prepared by serial dilutions of stock solutions with $50 \%$ 
methanol-water at the following concentrations: $6.25,12.5,25,50,100,250,500,1000 \mathrm{nM}$ of irinotecan; $6.25,12.5,25,50,100,250,500,1000 \mathrm{nM}$ of SN-38 and 3.12, 6.25, 12.5, 25, $50,100,250,500 \mathrm{nM}$ of SN-38 glucuronide. The standard and quality control solutions (QCs) were prepared by mixing $20 \mu \mathrm{l}$ of blank plasma, liver, kidney and fecal homogenates with each $20 \mu \mathrm{l}$ working solutions of CPT-11, SN-38 and SN-38 glucuronide in $1.5 \mathrm{ml}$ minieppendorf tubes. Quality control samples (QCs) were prepared at the following concentrations: 40, 200 and $1000 \mathrm{nM}$ for CPT-11, 40, 200 and $1000 \mathrm{nM}$ for SN-38 and 8, 40 and $200 \mathrm{nM}$ for SN-38 glucuronide. All sample solutions of rat plasma, feces, liver and kidney homogenates were also added with $20 \mu \mathrm{l}$ working solutions of CPT (100 nM). Then the solution is extracted with $360 \mu \mathrm{l}$ methanol-acetonitrile $(1: 1,[\mathrm{v} / \mathrm{v}])$ solution, vortex-mixed for $1 \mathrm{~min}$ and centrifuged at 15,500 rpm for $15 \mathrm{~min}$. The supernatants were transferred to another tube and evaporated to dryness under a steady stream of air at room temperature. The residue was reconstituted with $80 \mu \mathrm{l}$ of $50 \%$ aqueous methanolic solution and centrifuged again at 15,500 rpm for $15 \mathrm{~min}$. After centrifugation, $10 \mu \mathrm{l}$ of the supernatant was injected to UPLC-MS/MS system for analysis. In case of feces, we followed the same protocol except we added $360 \mu \mathrm{l}$ acetonitrile to extract irinotecan, $\mathrm{SN}-38$ and $\mathrm{SN}-38$ glucuronide. The linearity of each calibration curve was determined by plotting the ratio of the peak areas of analyte (Irinotecan/SN-38/SN-38 glucuronide) to internal standard (I.S.; CPT in 50\% methanol) in rat plasma, feces and homogenized liver and kidney. A leastsquare linear regression method $\left(1 / \mathrm{x}^{2}\right.$ weight) was used to determine the slope, intercept and correlation coefficient of linear regression method. The lower limit of quantification (LLOQ) was determined based on the signal-to-noise ratio of at least 10:1.

2.3.2. Accuracy and precision-The "intra-day" and "inter-day" precision and accuracy of the method were determined with quality control (QC) samples at three different concentrations (six injections for each concentration) on the same day or on three different days [34].

2.3.3. Extraction recovery and matrix effect-Extraction recovery of irinotecan, SN-38 and SN-38 glucuronide in different bio-matrices (plasma, feces, liver and kidney homogenate) was calculated by plotting the ratio of the peak areas of analyte (irinotecan/ SN-38/SN-38 glucuronide) to internal standard (CPT) in blank rat plasma/feces/liver or kidney homogenate spiked before extraction procedure divided by the ratio of the peak areas of analyte to internal standard for the same quantity of the respective compound spiked into extracted blank matrix. Similarly, matrix effects were calculated by dividing the ratio of the peak area of the compound and internal standard spiked into extracted blank matrix by the ratio of the peak area of same compound and internal standard in neat solution at the same concentration. All these experiments and evaluations were performed according to the recommended validation procedures reported by Matuszewski [34].

2.3.4. Stability-Short-term $\left(25^{\circ} \mathrm{C}\right.$ for $\left.4 \mathrm{~h}\right)$, post-processing $\left(20^{\circ} \mathrm{C}\right.$ for $\left.8 \mathrm{~h}\right)$, long-term $\left(-80^{\circ} \mathrm{C}\right.$ for 1 month) and three freeze-thaw cycle stabilities of irinotecan, $\mathrm{SN}-38$ and $\mathrm{SN}-38$ glucuronide were determined by analyzing three replicates of QC samples at three different concentrations. 


\subsection{In vivo rat pharmacokinetic study}

2.4.1. Animals-Male Wistar rats (6-10 weeks, Body weight between 250 to $280 \mathrm{~g}, \mathrm{n}=6$ ) were purchased from Harlan Laboratory (Indianapolis, IN). Rats were kept in an environmentally controlled room (temperature: $25 \pm 2{ }^{\circ} \mathrm{C}$, humidity: $50 \pm 5 \%, 12 \mathrm{~h}$ dark-light cycle) for at least 1 week before the experiments. The rats were fasted overnight before the day of the experiment.

2.4.2. Animal Experiment design-Irinotecan was administered at a dose of $5 \mathrm{mg} / \mathrm{kg}$ via intravenous injection through the tail vein. Blood samples (about $20-50 \mu \mathrm{L}$ ) were collected in heparinized tubes at $0,15,30,60,120,240,360,480$, and 1440 min postinjection, via tail snip with isoflurane as anesthetic. Urine samples were collected at 4, 8 and 24 hours; whereas feces were collected at 24 hour. Plasma samples were prepared and stored at $-80^{\circ} \mathrm{C}$ until analysis. The procedures were approved by the University of Houston's Institutional Animal Care and Uses Committee (IACUC).

2.4.3. Tissue homogenization-Liver and kidney were excised from the sacrificed rats and stored frozen at $-80^{\circ} \mathrm{C}$ in polypropylene tubes until homogenization. The frozen tissues were thawed, chopped and weighed at $4^{\circ} \mathrm{C}$. Accurately $50 \mathrm{mg}$ of chopped tissue was homogenized in $2 \mathrm{ml}$ of ice-cold homogenizing solution ( $\mathrm{pH}$ 7.4) containing $10 \mathrm{mM}$ potassium phosphate, $250 \mathrm{mM}$ sucrose and $1 \mathrm{mM}$ EDTA dehydrate with a polytron tissue homogenizer. Homogenization was paused $20 \mathrm{~s}$ after every $30 \mathrm{~s}$ of homogenization at a medium speed. The homogenization was repeated $3-4$ times until a uniform homogenate was obtained. Final tissue extract was stored at approximately $-80^{\circ} \mathrm{C}$ prior to analysis. The homogenizer probe was washed sequentially with water, methanol and water after every homogenization.

2.4.4. Sample preparation-Plasma, liver and kidney homogenate samples ( $20 \mu \mathrm{l})$ were spiked with $20 \mu \mathrm{l}$ of I.S. (CPT in $50 \%$ methanol, $100 \mathrm{nM}$ ) and vortexed for $1 \mathrm{~min}$ and then extracted with $360 \mu \mathrm{l}$ of $50 \%$ methanol in acetonitrile (1:1). All solutions were vortexed and centrifuged at 15,500 rpm for $15 \mathrm{~min}$. The supernatants were transferred to another tube and evaporated to dryness under a steady stream of air at room temperature. The residue was reconstituted with $80 \mu \mathrm{l}$ of $50 \%$ methanol in water (1:1) and centrifuged again at 15,500 rpm for $15 \mathrm{~min}$. After centrifugation, $10 \mu \mathrm{l}$ of the supernatant was injected to UPLC-MS/MS system for analysis. In case of fecal samples, they were first lyophilized to evaporate the moisture and then $1 \mathrm{~g}$ of dried feces was accurately weighed and homogenized with $10 \mathrm{ml}$ of homogenizing solution. The homogenized samples were centrifuged for $5 \mathrm{~min}$ to remove heavy particles and the supernatant was collected. Then, $20 \mu$ fecal homogenate solution was spiked with $20 \mu \mathrm{l}$ of I.S. (CPT in 50\% methanol, $100 \mathrm{nM}$ ) and vortexed for $1 \mathrm{~min}$ and extracted with 18 volume of acetonitrile for every volume of fecal homogenate. In case of urine, all the standard and samples were diluted 100 times with 50\% methanol-water solution, centrifuged at $15,500 \mathrm{rpm}$ for $15 \mathrm{~min}$ and $10 \mu \mathrm{l}$ of the supernatant was injected to UPLC-MS/MS system for quantification. The density of the blood is treated as $1 \mathrm{~g} / \mathrm{mL}$ in the tissue distribution study. 
2.4.5. Preparation of standards, quality controls and sample solutionsCalibration standards and quality control (QC) samples were prepared as described in section 2.3.1.

2.4.6. Pharmacokinetic parameter calculation-The pharmacokinetic parameters of irinotecan, SN-38 and SN-38 glucuronide were calculated by the non-compartmental method, using Phoenix WinNonLin (Pharsight Corporation, Mountain View, California) program.

\section{Results and Discussion}

\subsection{Optimization of the UPLC-MS/MS condition}

Different combinations of mobile and stationary phases were employed to enhance the sensitivity of detection of irinotecan, SN-38 and SN-38 glucuronide. Acetonitrile, methanol, $0.1-5 \%$ formic acid in acetonitrile, and $0.1-5 \%$ formic acid in methanol as organic phase and $1-2.5 \mathrm{mM}$ ammonium acetate in water, $0.05-1 \%$ formic acid in water as aqueous phase were tested as potential mobile phases. We tested both $\mathrm{C} 8$ and $\mathrm{C} 18$ column as stationary phases to resolve irinotecan, $\mathrm{SN}-38$ and $\mathrm{SN}-38$ glucuronide. Based on the intensity of the signal, signal-noise ratio and shape of the peak, $0.1 \%$ formic acid in water, $100 \%$ acetonitrile and C18 column were found to be the optimal aqueous mobile phase, organic mobile phase and stationary phase, respectively. Apart from that, it has been found that column temperature at $60^{\circ} \mathrm{C}$ and the flow rate of $0.45 \mathrm{ml} / \mathrm{min}$ produced sharp and symmetrical peaks (Fig $1 \mathrm{~A}$ ). For MS/MS analysis, both positive and negative scan mode were employed to analyze irinotecan, $\mathrm{SN}-38$ and $\mathrm{SN}-38$ glucuronide. A representative MS/MS spectrum of irinotecan, $\mathrm{SN}-38$ and SN-38 glucuronide is shown in figure $1 \mathrm{~B}, 1 \mathrm{C}$ and $1 \mathrm{D}$, respectively. Based on the intensity of the analytes, positive scan mode was found to be more sensitive compared to the negative scan mode. To improve the specificity, multiple reactions monitoring (MRM) scan type was used. The MRM transitions from precursor ions to product ions were optimized as $\mathrm{m} / \mathrm{z} 587.6 \rightarrow 124.04$ for irinotecan, 393.1 $\rightarrow 349.06$ for SN-38, $569.05 \rightarrow 393$ for SN-38 glucuronide and $349 \rightarrow 305$ for CPT (IS), based on their most abundant precursor ions and corresponding product ions (Fig. $1 \mathrm{~B}, 1 \mathrm{C}$, $1 \mathrm{D}$ and $1 \mathrm{E})$. Additional compound-dependent parameters in MRM mode for irinotecan, SN-38, SN-38 glucuronide and CPT were summarized in Table 1. The main working parameters for mass spectrum were used in the QTRAP 5500 system as follows: ion-spray voltage, $5.5 \mathrm{kV}$; temperature, $500^{\circ} \mathrm{C}$; curtain gas, $20 \mathrm{psig}$; gas $1,20 \mathrm{psig}$; gas $2 ; 20 \mathrm{psig}$, collision gas, medium.

\subsection{Method Validation}

3.2.1. Linearity and sensitivity-Method validation was conducted using blank rat plasma, untreated feces, liver and kidney tissue samples. In plasma, the standard curves of irinotecan, $\mathrm{SN}-38$ and $\mathrm{SN}-38$ glucuronide were linear in the concentration range of $4.88-$ $10000 \mathrm{nM}, 4.88-10000 \mathrm{nM}$, and $6.25-2000 \mathrm{nM} \mathrm{SN}-38$ glucuronide, respectively $\left(\mathrm{R}^{2}>\right.$ 0.99). In case of feces samples, the standard curves of irinotecan, SN-38, SN-38 glucuronide were linear in the concentration range of $39-5000 \mathrm{nM}, 39-5000 \mathrm{nM}$, and $4.88-1250 \mathrm{nM}$ SN-38 glucuronide, respectively $\left(\mathrm{R}^{2}>0.99\right)$. The assay also exhibited excellent linear 
response over selected concentration range of $48.8-6250 \mathrm{nM}, 48.8-6250 \mathrm{nM}$ and $9.8-$ $1250 \mathrm{nM}$ for irinotecan, SN-38 and SN-38 glucuronide, respectively in liver and kidney homogenates $\left(\mathrm{R}^{2}>0.99\right)$. The lower limit of detection (LLOD) of irinotecan and SN-38 was determined to be $2.44 \mathrm{nM}, 19.5 \mathrm{nM}$ and $24.44 \mathrm{nM}$ in plasma, feces, liver and kidney homogenate, respectively. In case of SN-38 glucuronide, LLOD is $6.25 \mathrm{nM}, 2.44 \mathrm{nM}$ and 4.9 $\mathrm{nM}$ in plasma, feces, liver and kidney homogenate, respectively. However, the LLOD values of irinotecan, $\mathrm{SN}-38$ and SN-38 glucuronide in rat plasma weren't comparable with the values (116 pg/ml for irinotecan, $58 \mathrm{pg} / \mathrm{ml}$ for SN-38 and $105 \mathrm{pg} / \mathrm{ml}$ for SN-38 glucuronide) reported by Marangon et al [31]., which might be explained by the differences of nature of sample analyzed based on species (rat vs human).

3.2.2. Accuracy and precision-Accuracy, intra-day and inter-day precision were determined by running six replicates of $\mathrm{QC}$ samples at three different concentration levels (low, medium, high) of irinotecan, SN-38 and SN-38 glucuronide in blank rat plasma, feces, liver and kidney tissues. The precision and accuracy of these measurements were shown in Table 2. These results demonstrated that the precision and accuracy values were in the acceptance range $(<15 \%)$ according to FDA guidance. Unlike LLOD values, the precision and accuracy values were quite comparable with the values reported by Marangon et al [31].

3.2.3. Recovery, matrix effect and stability-The mean extraction recoveries determined using three replicates of QC samples at three concentration levels (the same concentrations as QC sample) in rat plasma, feces, liver and kidney tissues were shown in Table 2, 3, 4 and 5, respectively. 50\% methanol-acetonitrile and 100\% acetonitrile were used as protein precipitating solvent to extract irinotecan, $\mathrm{SN}-38$ and SN-38 glucuronide from blank rat plasma, liver, kidney and feces. The result showed that the recoveries were not less than $85 \%$ for all the analytes at three different concentrations in plasma and feces. In comparison to human plasma (44-84\%), the recoveries of irinotecan and its metabolites in rat plasma were quite higher (95-114\%) [31].

Similarly, to test if the matrix effects have any impact on the UPLC-MS analysis of irinotecan, SN-38 and SN-38 glucuronide, the relative peak areas of the analyte spiking in the evaporated blank plasma, feces, liver and kidney tissues at three different concentration levels (low, medium and high) were compared to similarly prepared standard solutions. As shown in the table 2, 3, 4 and 5, no measurable matrix effect was observed.

Stabilities of irinotecan, SN-38 and SN-38 glucuronide in plasma were evaluated by analyzing triplicates of QC samples at three different concentrations following storage at $25^{\circ} \mathrm{C}$ for $4 \mathrm{~h}$, at $-80^{\circ} \mathrm{C}$ for 3 days, and after going through three freeze-thaw cycles $\left(-80^{\circ} \mathrm{C}\right.$ and $25^{\circ} \mathrm{C}$ ). All the samples showed $85-115 \%$ recoveries after various stability tests.

\subsection{Application to the method to determine the plasma, urine and feces concentration of Irinotecan in rats after i.v. administration}

The validated method was utilized to determine irinotecan, SN-38 and SN-38 glucuronide content in plasma, feces and urine after intravenous administration of $5 \mathrm{mg} / \mathrm{kg}$ irinotecan in male wistar rats. Mean plasma concentration of irinotecan, SN-38 and SN-38 glucuronide as a function of time after i.v. administration of irinotecan is shown in fig 2 . The $\mathrm{C}_{\max }$ of 
irinotecan, $\mathrm{SN}-38$ and $\mathrm{SN}-38$ glucuronide are $6.02 \pm 0.19 \mu \mathrm{M}, 2.13 \pm 0.70 \mu \mathrm{M}$ and 0.08 $\pm 0.01 \mu \mathrm{M}$, respectively; whereas the $\mathrm{T}_{\max }$ and AUC of irinotecan, $\mathrm{SN}-38$ and $\mathrm{SN}-38$ glucuronide are $33.75 \mathrm{~min}, 26.25 \mathrm{~min}, 100 \mathrm{~min}$ and $15.72 \mathrm{~h} \mu \mathrm{M}, 18.53 \mathrm{~h} \mu \mathrm{M}$ and $0.70 \mathrm{~h} \mu \mathrm{M}$, respectively. The plasma concentration-time profile of irinotecan continued to exhibit multi exponential phases denoting rapid distribution and slower elimination. In case of urinary excretion it was found that in comparison to $\mathrm{SN}-38(16.50 \mu \mathrm{M})$ and its glucuronidated form $(11.47 \mu \mathrm{M})$, irinotecan $(67.49 \mu \mathrm{M})$ predominantly excreted in unchanged form after 4 hours (fig 3). However, after 24 hours, the concentration of irinotecan $(6.43 \mu \mathrm{M})$ and $\mathrm{SN}-38$ glucuronide $(6.07 \mu \mathrm{M})$ in urine is quite comparable. After analyzing the feces we found that, after 24 hour (fig 4), fecal content of irinotecan $(818.35 \mu \mathrm{g} / \mathrm{g})$ and SN-38 $(423.95 \mu \mathrm{g} / \mathrm{g})$ is much higher than the fecal content of SN-38 glucuronide $(9.60 \mu \mathrm{g} / \mathrm{g})$. Low fecal excretion of $\mathrm{SN}-38$ glucuronide might be ascribed to the hydrolysis of SN-38 glucuronide by bacterial $\beta$ glucuronidase in feces which was supported by different reports $[35,36]$.

\section{Conclusion}

In this study, we developed and validated a rapid, sensitive and specific UPLC-MS/MS method for quantifying irinotecan, SN-38 and SN-38 glucuronide simultaneously in rat plasma, feces, urine, liver, kidney, and the validated method was successfully applied to the in vivo pharmacokinetic studies of irinotecan in rats. This is the first UPLC-MS/MS method that allows us to simultaneously quantify irinotecan, its active metabolite SN-38 and SN-38glucuronide in different bio-matrices (plasma, urine and feces) as well as in different tissues (liver and kidney). Apart from that, this method has certain advantages such as high sensitivity (nM), short analysis time (4.5 min), good recovery with negligible matrix effect. This method can also be extrapolated to clinical studies owing to its high sensitivity and small sample volume requirement; although full validation of the method in human matrices is required prior to support any kind of clinical studies.

\section{References}

1. Tournigand C, et al. FOLFIRI followed by FOLFOX6 or the reverse sequence in advanced colorectal cancer: a randomized GERCOR study. J Clin Oncol. 2004; 22(2):229-237. [PubMed: 14657227]

2. Akhtar R, et al. Current status of pharmacological treatment of colorectal cancer. World J Gastrointest Oncol. 2014; 6(6):177-183. [PubMed: 24936228]

3. Ohno R, et al. An early phase II study of CPT-11: a new derivative of camptothecin, for the treatment of leukemia and lymphoma. J Clin Oncol. 1990; 8(11):1907-1912. [PubMed: 2230878]

4. Fukuoka M, et al. A phase II study of CPT-11, a new derivative of camptothecin, for previously untreated non-small-cell lung cancer. J Clin Oncol. 1992; 10(1):16-20. [PubMed: 1309380]

5. Shimada Y, et al. Phase II study of CPT-11, a new camptothecin derivative, in metastatic colorectal cancer. CPT-11 Gastrointestinal Cancer Study Group. J Clin Oncol. 1993; 11(5):909-913. [PubMed: 8487053]

6. Wall ME, W MC, Cook CE, Palmer KH, McPhail AT, Sim GA. Plant antitumor agents. I. The isolation and structure of camptothecine, a novel alkaloidal leukemia and tumor inhibitor from Camptotheca acuminata. Journal of the American Chemical Society. 1966; 88(16):3888-3890.

7. Sawada S, et al. Synthesis and antitumor activity of 20(S)-camptothecin derivatives: carbamatelinked, water-soluble derivatives of 7-ethyl-10-hydroxycamptothecin. Chem Pharm Bull (Tokyo). 1991; 39(6):1446-1450. [PubMed: 1934165] 
8. Rothenberg ML, et al. Phase I and pharmacokinetic trial of weekly CPT-11. J Clin Oncol. 1993; 11(11):2194-2204. [PubMed: 8229134]

9. Mathijssen RH, et al. Clinical pharmacokinetics and metabolism of irinotecan (CPT-11). Clin Cancer Res. 2001; 7(8):2182-2194. [PubMed: 11489791]

10. Redinbo MR, et al. Crystal structures of human topoisomerase I in covalent and noncovalent complexes with DNA. Science. 1998; 279(5356):1504-1513. [PubMed: 9488644]

11. Stewart L, et al. A model for the mechanism of human topoisomerase I. Science. 1998; 279(5356): 1534-1541. [PubMed: 9488652]

12. Gupta E, et al. Metabolic fate of irinotecan in humans: correlation of glucuronidation with diarrhea. Cancer Res. 1994; 54(14):3723-3725. [PubMed: 8033091]

13. Hecht JR. Gastrointestinal toxicity or irinotecan. Oncology (Williston Park). 1998; 12(8 Suppl 6): 72-78. [PubMed: 9726096]

14. Sugiyama Y, Kato Y, Chu X. Multiplicity of biliary excretion mechanisms for the camptothecin derivative irinotecan (CPT-11), its metabolite SN-38, and its glucuronide: role of canalicular multispecific organic anion transporter and P-glycoprotein. Cancer Chemother Pharmacol. 1998; 42(Suppl):S44-S49. [PubMed: 9750028]

15. Itoh T, et al. Biliary excretion of irinotecan and its metabolites. J Pharm Pharm Sci. 2004; 7(1):1318. [PubMed: 15144730]

16. Gupta E, et al. Pharmacokinetic modulation of irinotecan and metabolites by cyclosporin A. Cancer Res. 1996; 56(6):1309-1314. [PubMed: 8640819]

17. Ciotti M, et al. Glucuronidation of 7-ethyl-10-hydroxycamptothecin (SN-38) by the human UDPglucuronosyltransferases encoded at the UGT1 locus. Biochem Biophys Res Commun. 1999; 260(1):199-202. [PubMed: 10381366]

18. Gagne JF, et al. Common human UGT1A polymorphisms and the altered metabolism of irinotecan active metabolite 7-ethyl-10-hydroxycamptothecin (SN-38). Mol Pharmacol. 2002; 62(3):608617. [PubMed: 12181437]

19. Catimel G, et al. Phase I and pharmacokinetic study of irinotecan (CPT-11) administered daily for three consecutive days every three weeks in patients with advanced solid tumors. Ann Oncol. 1995; 6(2):133-140. [PubMed: 7786821]

20. Wallace BD, et al. Alleviating cancer drug toxicity by inhibiting a bacterial enzyme. Science. 2010; 330(6005):831-835. [PubMed: 21051639]

21. Kaneda N, Yokokura T. Nonlinear pharmacokinetics of CPT-11 in rats. Cancer Res. 1990; 50(6): 1721-1725. [PubMed: 2306726]

22. Slichenmyer WJ, et al. The current status of camptothecin analogues as antitumor agents. J Natl Cancer Inst. 1993; 85(4):271-291. [PubMed: 8381186]

23. Sano K, et al. Simple non-ion-paired high-performance liquid chromatographic method for simultaneous quantitation of carboxylate and lactone forms of 14 new camptothecin derivatives. $\mathrm{J}$ Chromatogr B Analyt Technol Biomed Life Sci. 2003; 795(1):25-34.

24. Boyd G, et al. High-performance liquid chromatographic technique for the simultaneous determination of lactone and hydroxy acid forms of camptothecin and SN-38 in tissue culture media and cancer cells. Anal Biochem. 2001; 297(1):15-24. [PubMed: 11567523]

25. Hu ZP, et al. Simultaneous determination of irinotecan (CPT-11) and SN-38 in tissue culture media and cancer cells by high performance liquid chromatography: application to cellular metabolism and accumulation studies. J Chromatogr B Analyt Technol Biomed Life Sci. 2007; 850(1-2):575580.

26. Gravel E, et al. Fluorescence detection combined with either HPLC or HPTLC for pharmaceutical quality control in a hospital chemotherapy production unit: application to camptothecin derivatives. J Pharm Biomed Anal. 2005; 39(3-4):581-586. [PubMed: 16023817]

27. Yang X, et al. Simultaneous determination of the lactone and carboxylate forms of irinotecan (CPT-11) and its active metabolite SN-38 by high-performance liquid chromatography: application to plasma pharmacokinetic studies in the rat. J Chromatogr B Analyt Technol Biomed Life Sci. 2005; 821(2):221-228. 
28. Corona G, et al. Fast liquid chromatography-tandem mass spectrometry method for routine assessment of irinotecan metabolic phenotype. Ther Drug Monit. 2010; 32(5):638-646. [PubMed: 20683392]

29. Ahn G, et al. A rapid, simple and reliable HPLC-triple quadrupole tandem mass spectrometer method for a simultaneous quantification of irinotecan and its active metabolite 7-ethyl-10hydroxycamptothecin (SN38) in mouse plasma. Biomed Chromatogr. 2014; 28(7):919-922. [PubMed: 24458571]

30. Park DJ, et al. Determination of irinotecan and its metabolite SN-38 in rabbit plasma and tumors using a validated method of tandem mass spectrometry coupled with liquid chromatography. $\mathrm{J}$ Chromatogr B Analyt Technol Biomed Life Sci. 2014; 962:147-152.

31. Marangon E, et al. Development and validation of a high-performance liquid chromatographytandem mass spectrometry method for the simultaneous determination of irinotecan and its main metabolites in human plasma and its application in a clinical pharmacokinetic study. PLoS One. 2015; 10(2):e0118194. [PubMed: 25689738]

32. Bardin S, et al. Liquid chromatographic-tandem mass spectrometric assay for the simultaneous quantification of Camptosar and its metabolite SN-38 in mouse plasma and tissues. J Chromatogr A. 2005; 1073(1-2):249-255. [PubMed: 15909526]

33. Goldwirt L, et al. A new UPLC-MS/MS method for the determination of irinotecan and 7-ethyl-10hydroxycamptothecin ( $\mathrm{SN}-38)$ in mice: application to plasma and brain pharmacokinetics. J Pharm Biomed Anal. 2012; 66:325-333. [PubMed: 22551773]

34. Matuszewski BK, Constanzer ML, Chavez-Eng CM. Strategies for the assessment of matrix effect in quantitative bioanalytical methods based on HPLC-MS/MS. Anal Chem. 2003; 75(13):30193030. [PubMed: 12964746]

35. Sparreboom A, et al. Irinotecan (CPT-11) metabolism and disposition in cancer patients. Clin Cancer Res. 1998; 4(11):2747-2754. [PubMed: 9829738]

36. Slatter JG, et al. Pharmacokinetics, metabolism, and excretion of irinotecan (CPT-11) following I.V. infusion of [(14)C]CPT-11 in cancer patients. Drug Metab Dispos. 2000; 28(4):423-433. [PubMed: 10725311] 


\section{HIGHLIGHTS}

1. Developed a UPLC-MS/MS method to simultaneously quantitate irinotecan, SN-38 and SN-38 glucuronide in different bio-matrices (plasma, bile, feces, and urine) and organs (liver and kidney).

2. Validated the sensitive and robust LC-MS method with respect to precision and accuracy in different bio-matrices and organs.

3. Showed in comparison to SN-38 and its glucuronidated form, irinotecan excreted predominantly as unchanged form through urine.

4. Found the fecal excretion of SN-38 glucuronide is negligible with respect to irinotecan and $\mathrm{SN}-38$. 
A

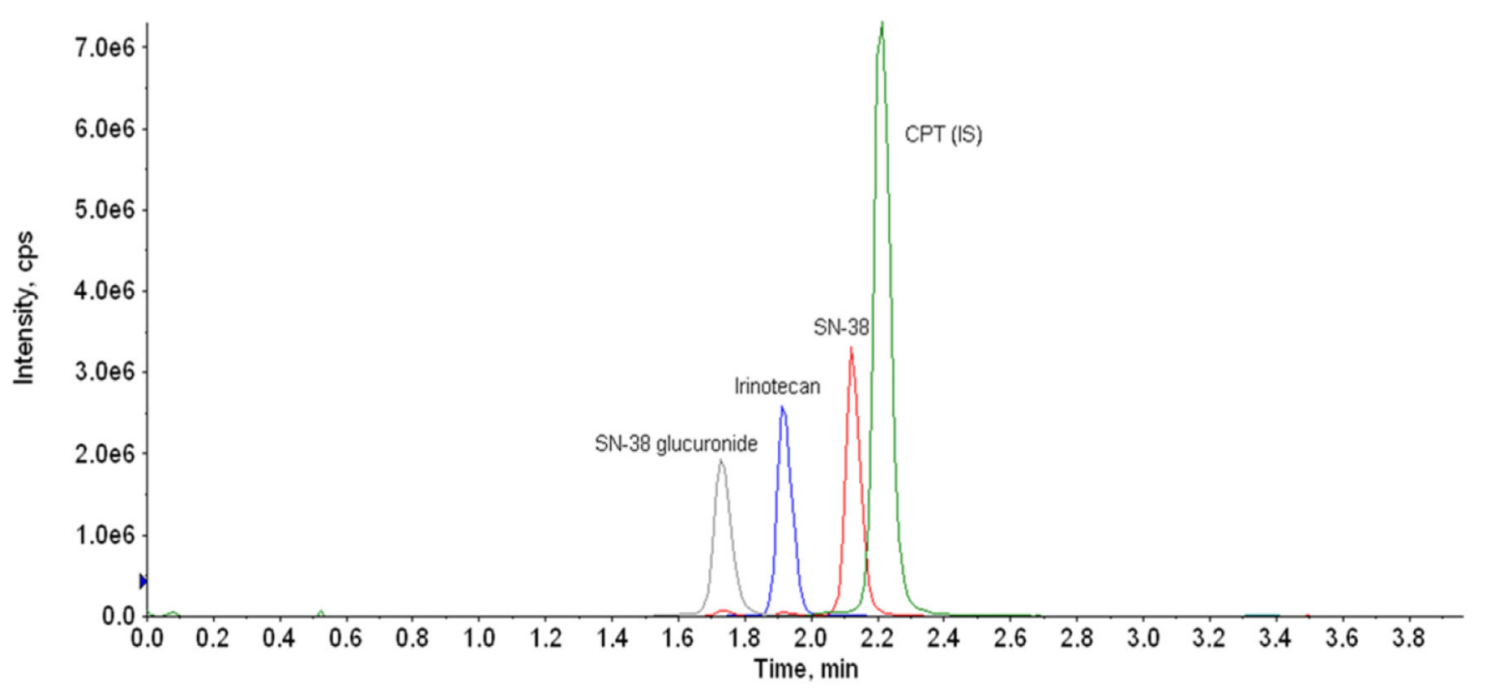

B Irinoteca

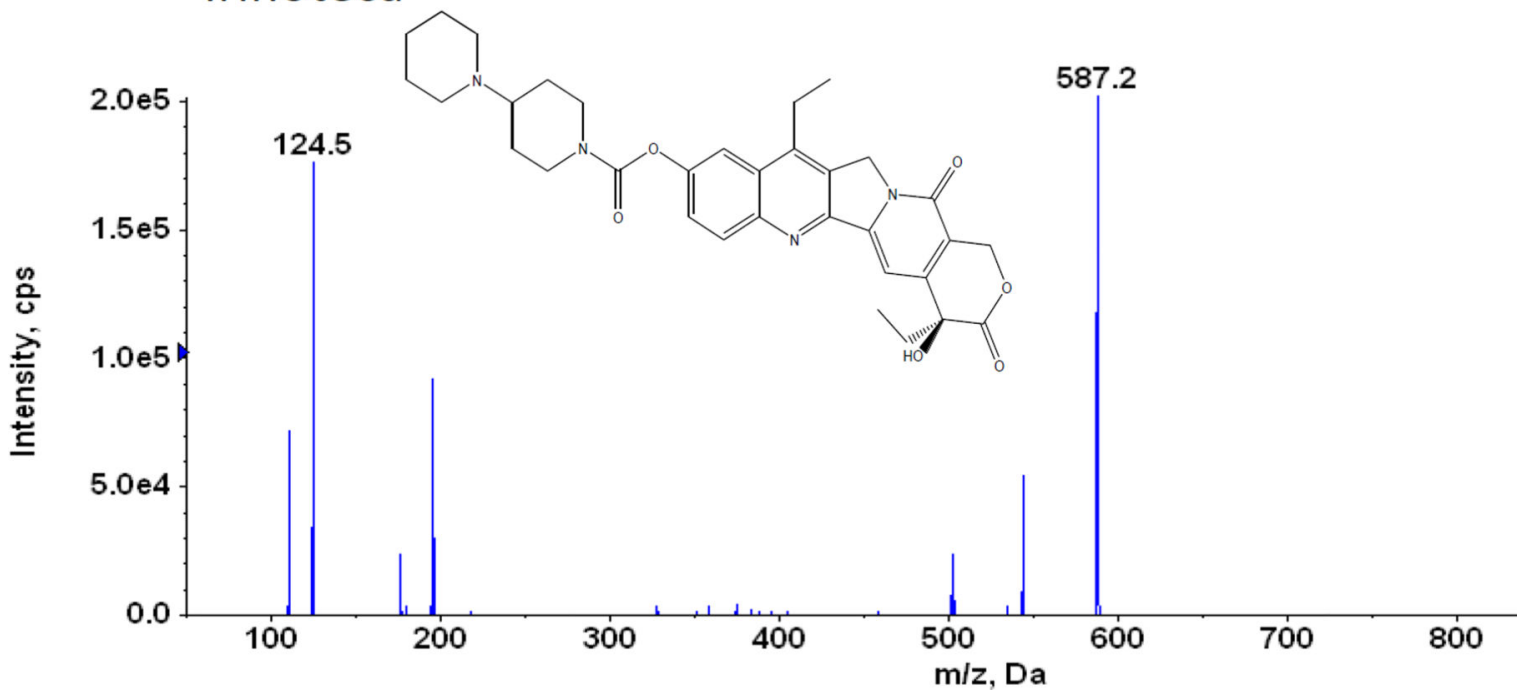



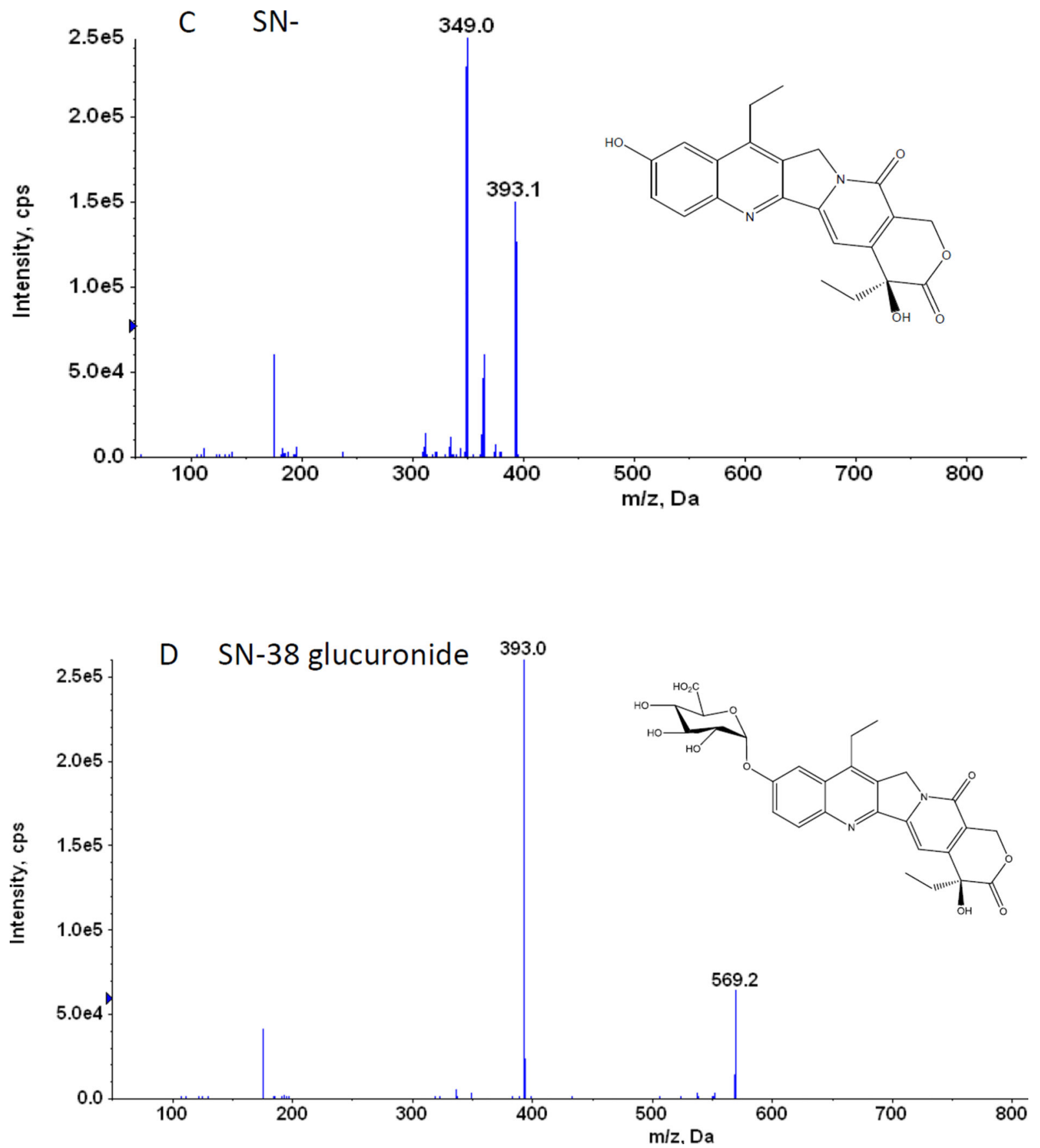
E CPT (IS)

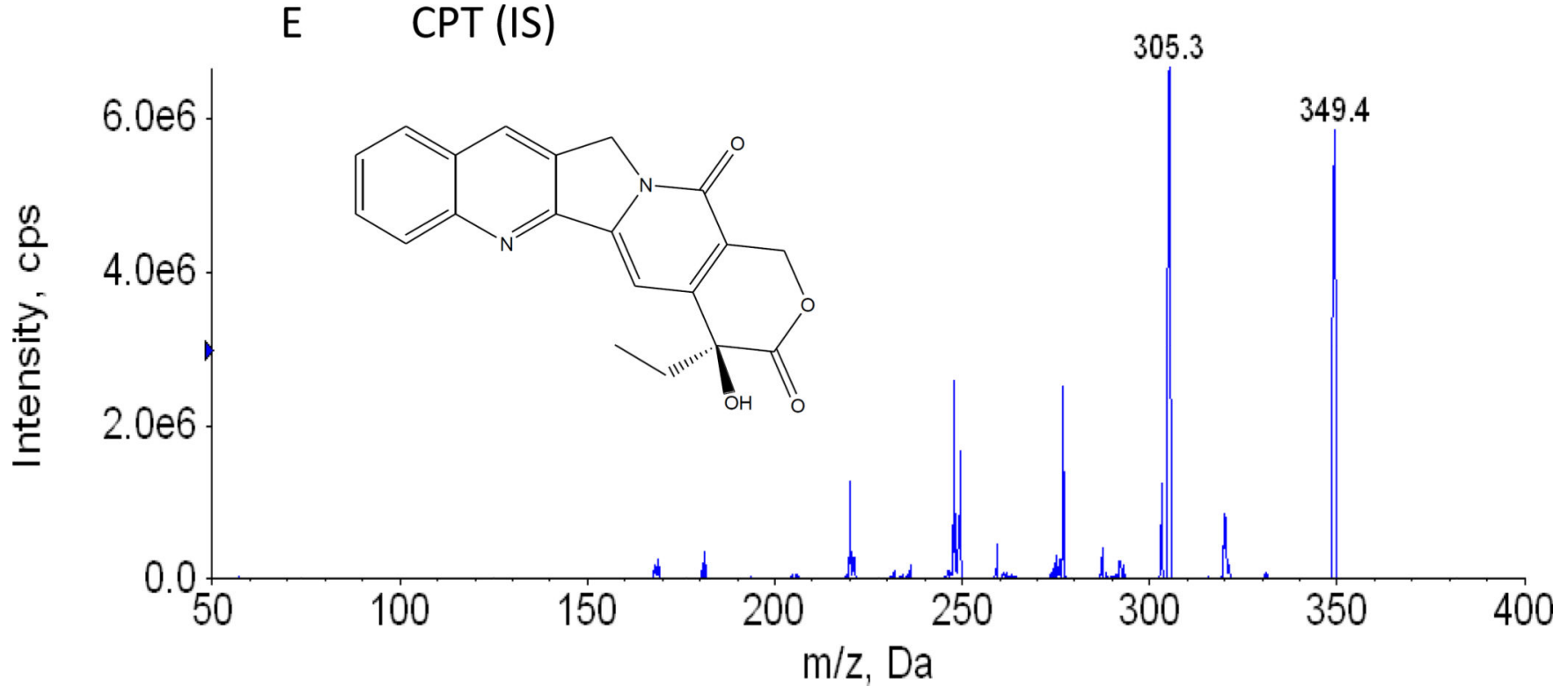

Figure 1.

A. Representative UPLC chromatogram of irinotecan, SN-38, SN-38 glucuronide and CPT (IS)

B. Chemical structures and representative MS/MS Chromatogram of irinotecan

C. Chemical structures and representative MS/MS Chromatogram of SN-38

D. Chemical structures and representative MS/MS Chromatogram of SN-38 glucuronide

E. Chemical structures and representative MS/MS Chromatogram of CPT (IS) 

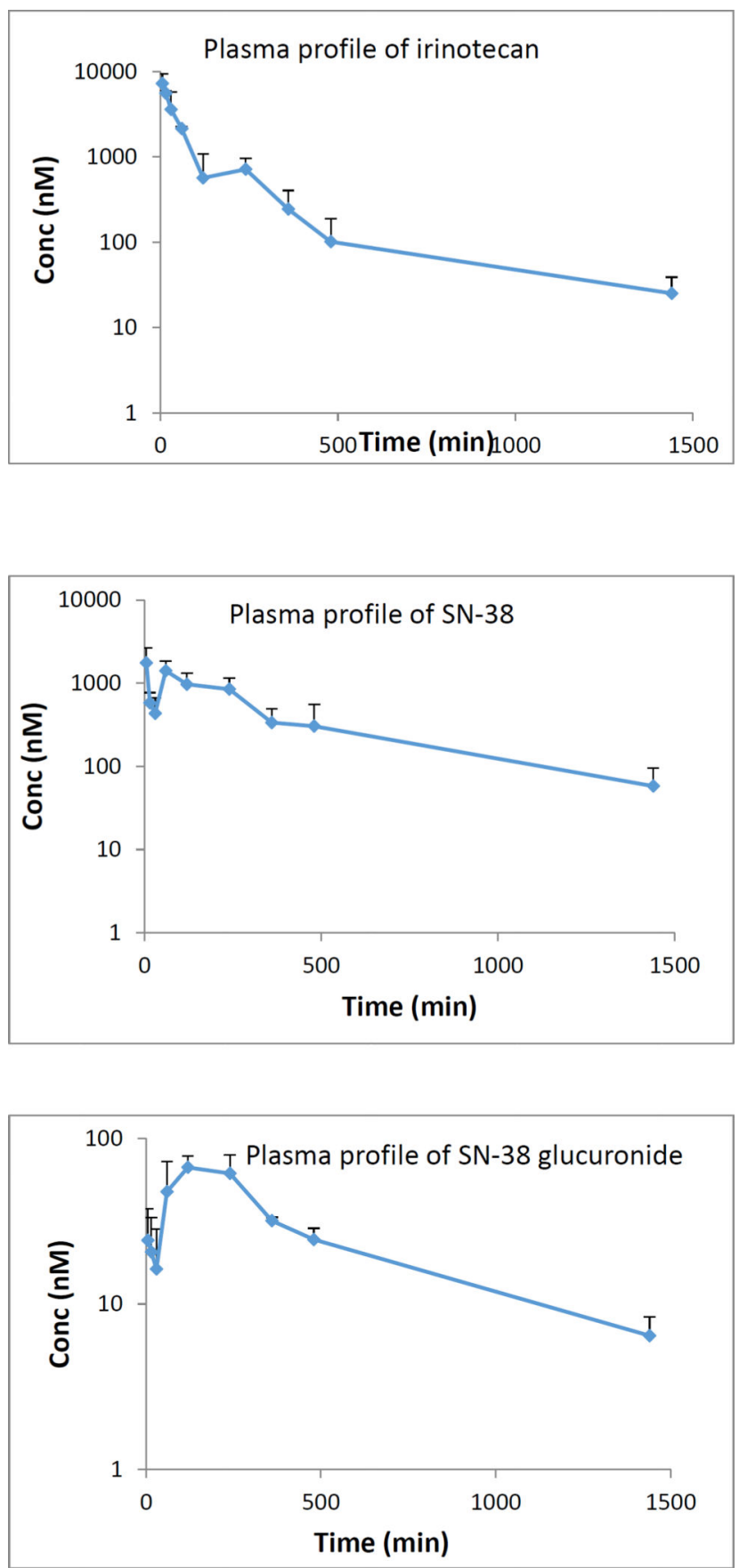

Figure 2.

Plasma profile of irinotecan, SN-38 and SN-38 glucuronide after intravenous administration of $5 \mathrm{mg} / \mathrm{kg}$ irinotecan 

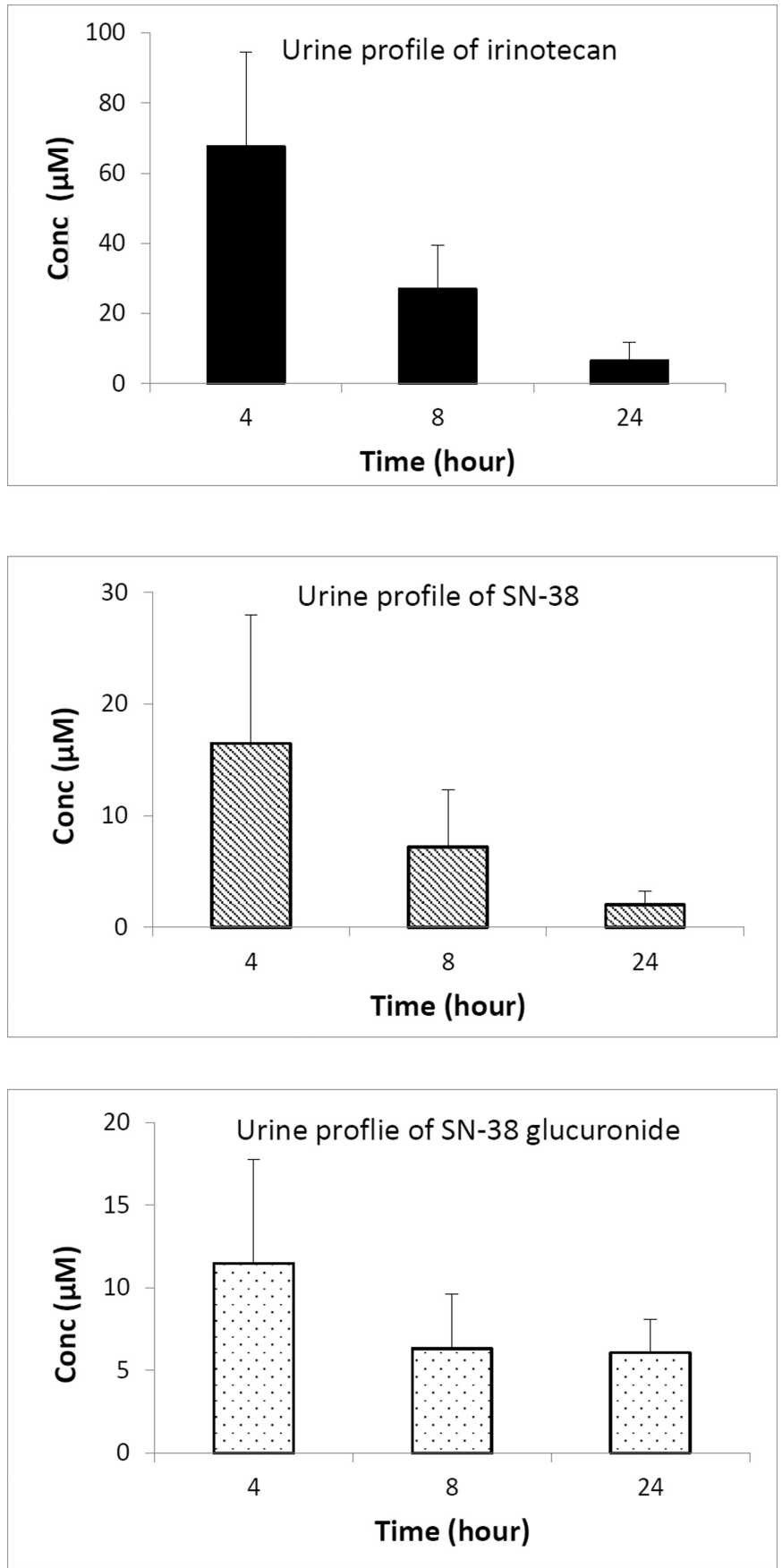

Figure 3.

Urine profile of irinotecan, $\mathrm{SN}-38$ and $\mathrm{SN}-38$ glucuronide after intravenous administration of $5 \mathrm{mg} / \mathrm{kg}$ irinotecan 


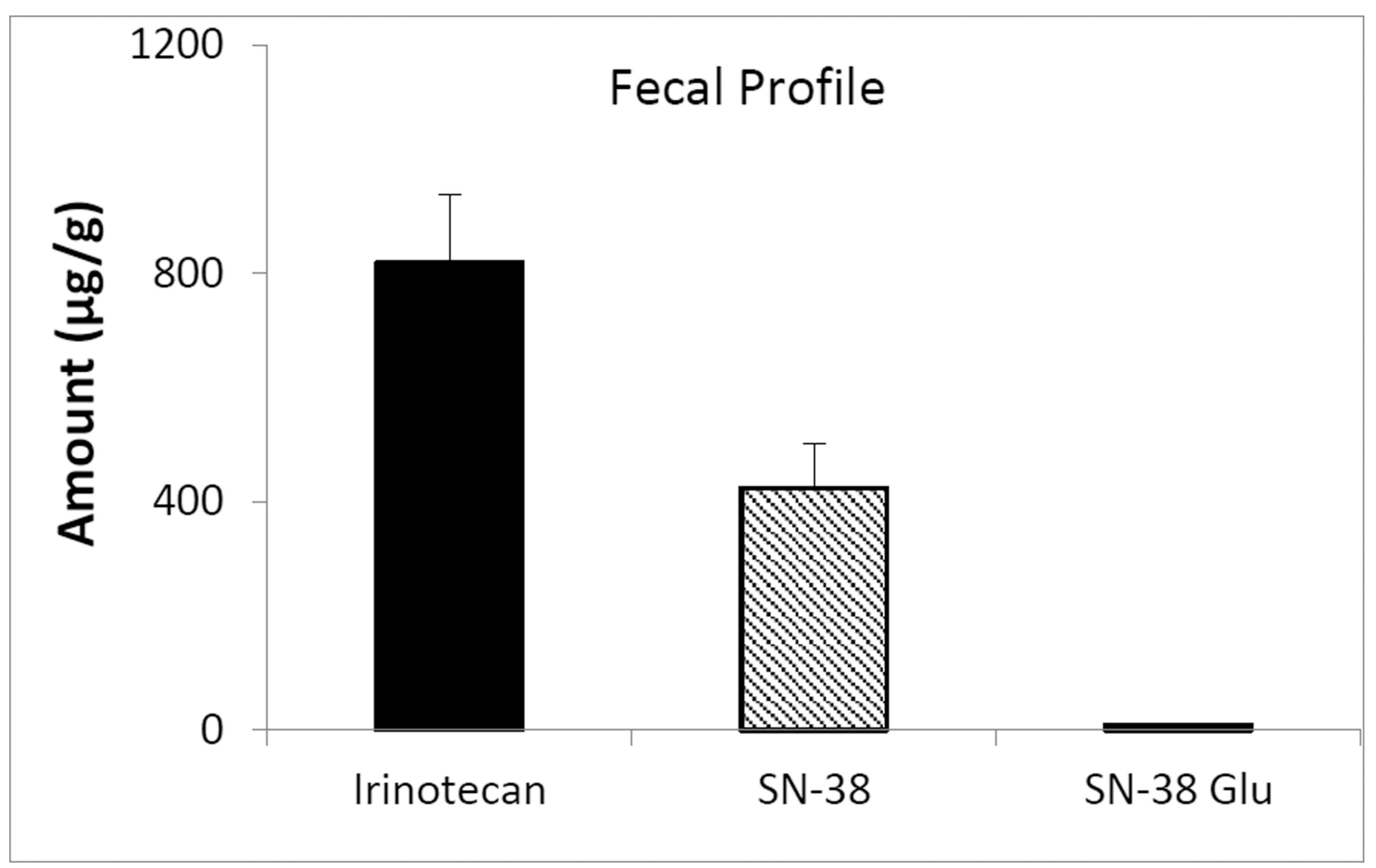

Figure 4.

Fecal profile of irinotecan, SN-38 and SN-38 glucuronide in feces collected for 24 hours, after intravenous administration of $5 \mathrm{mg} / \mathrm{kg}$ irinotecan 


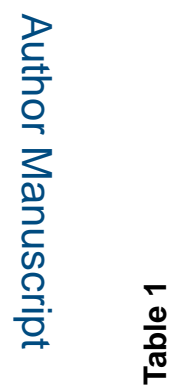

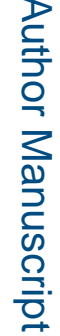

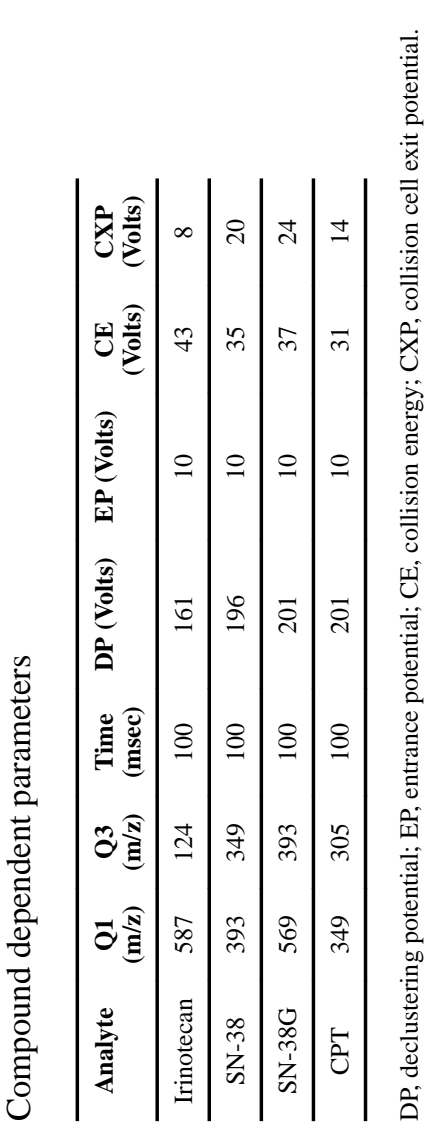

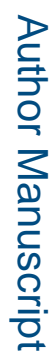

$$
\text { 送 }
$$




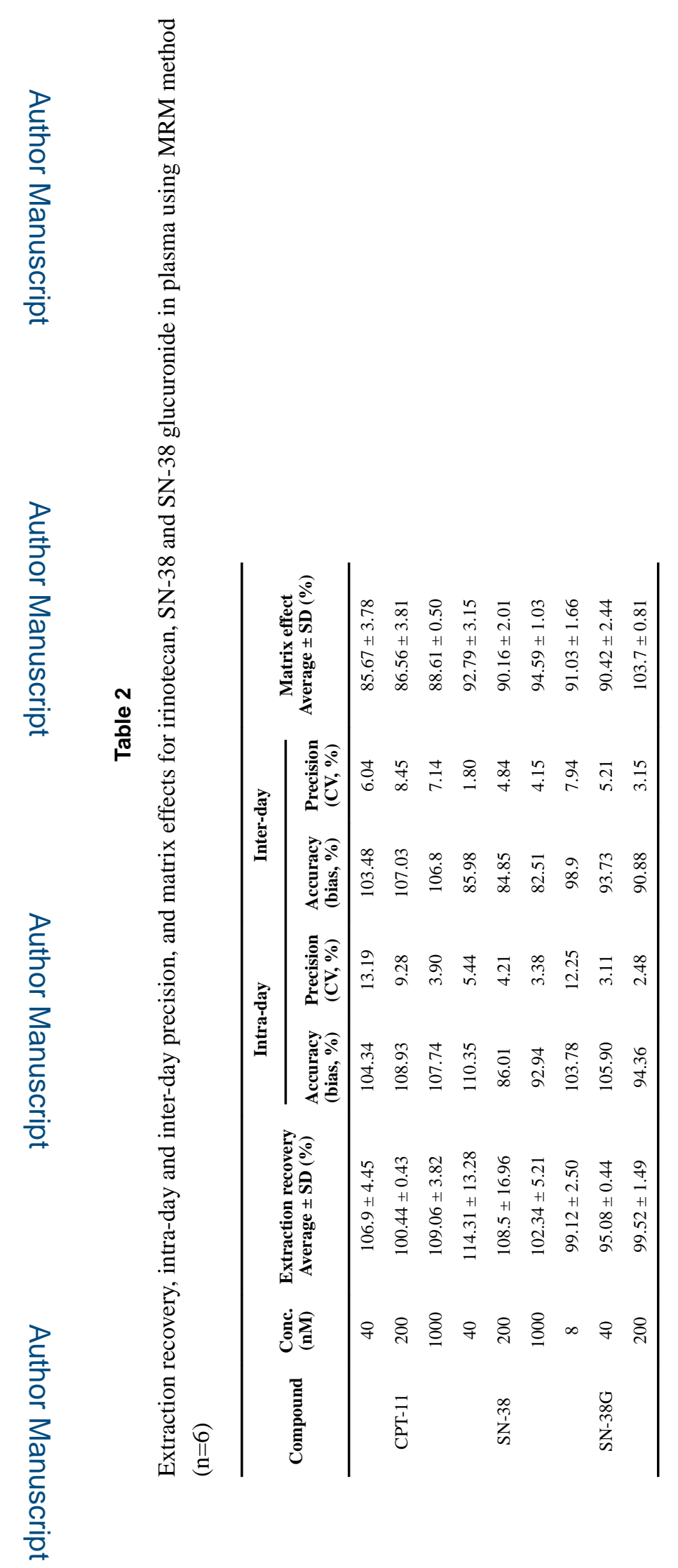




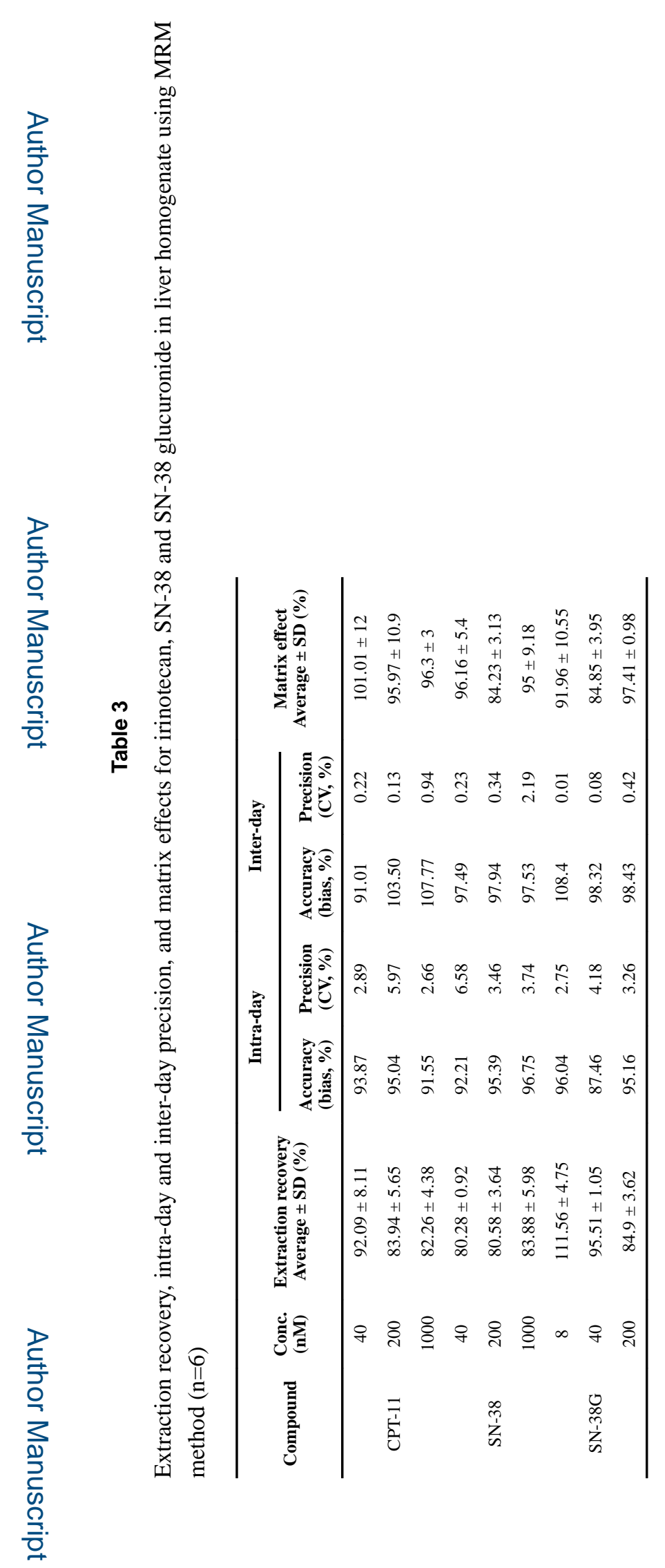




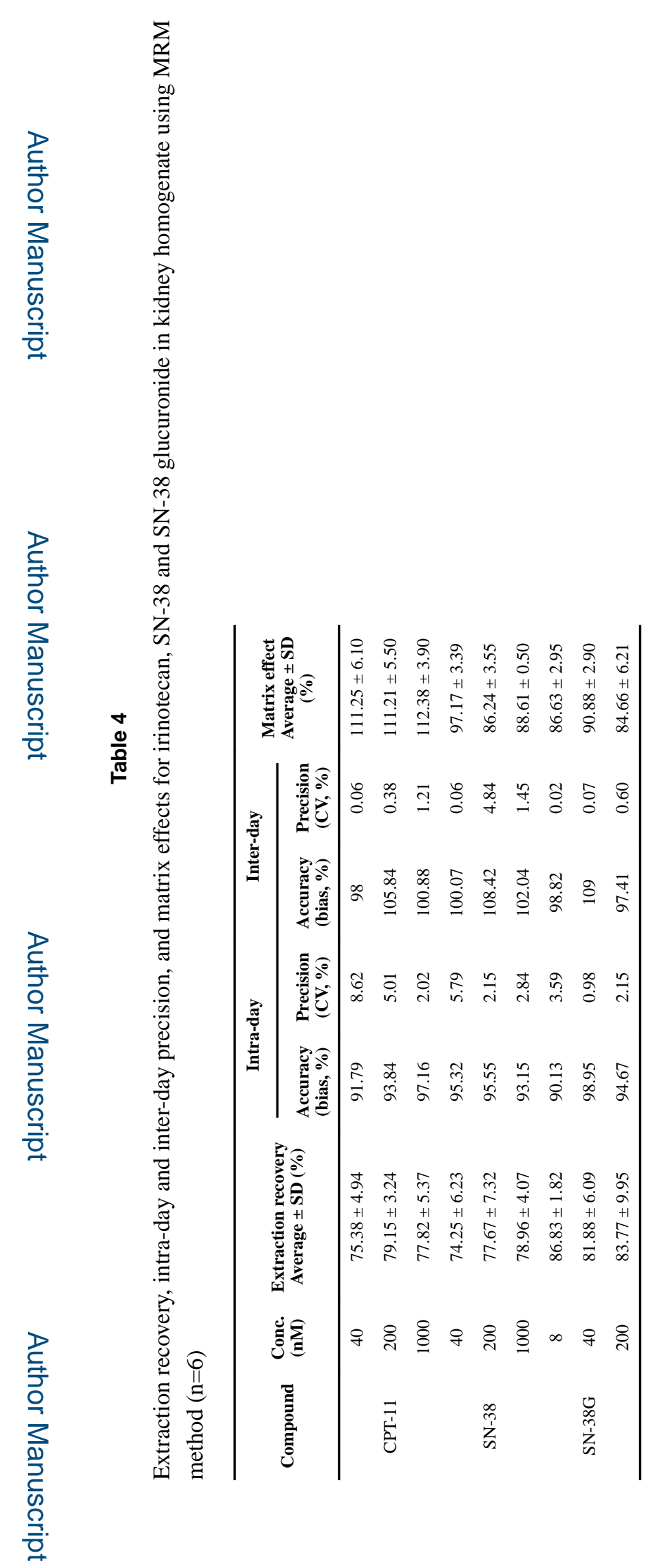




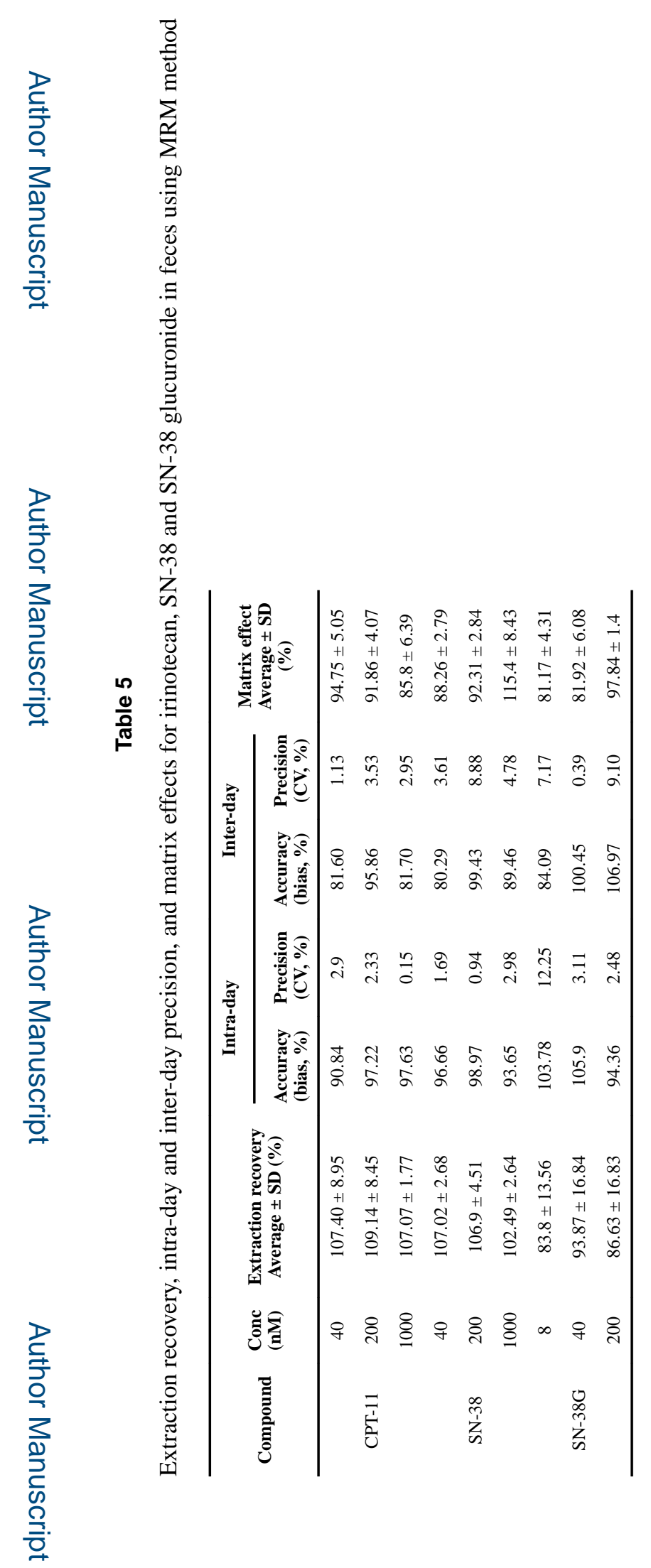

J Chromatogr B Analyt Technol Biomed Life Sci. Author manuscript; available in PMC 2017 March 15. 